2. A. Kolmogoroff, Une contribution de l'étude de la convergence des series de Fourier, Fund. Math. vol. 5 (1924) pp. 96-97.

3. J. E. Littlewood and R. E. A. C. Paley, Theorems on Fourier series and power series, J. London Math. Soc. vol. 6 (1931) pp. 230-233.

4. - Theorems on Fourier series and power series, (2), Proc. London Math. Soc. (2) vol. 42 (1937) pp. 52-89.

5. J. Marcinkiewicz and A. Zygmund, $A$ theorem of Lusin, Duke Math. J. vol. 4 (1938) pp. 473-485.

6. G. Sunouchi, On the strong summability of Fourier series, Proc. Imp. Acad. Tokyo vol. 19 (1943) pp. 420-423.

7. Z. Zalcwasser, Sur la sommabilité des séries de Fourier, Studia Mathematica vol. 6 (1936) pp. 82-88.

8. A. Zygmund, Sur la caractère de divergence des séries orthogonales, Mathematica vol. 9 (1935) pp. 86-88.

9. - Proof of a theorem of Paley, Proc. Cambridge Philos. Soc. vol. 34 (1938) pp. 125-133.

10. - On the convergence and summability of power series on the circle of convergence (1), Fund. Math. vol. 30 (1938) pp. 170-196.

TOHOKU IMPERIAL UNIVERSITY

\title{
ON THEOREMS OF M. RIESZ AND ZYGMUND
}

\section{A. P. CALDERón}

Several proofs have been given of the results of M. Riesz and Zygmund.

(a) The conjugate of the Fourier series of a function $f(x)$ of $L^{p}, p>1$, is Fourier series of a function $\bar{f}(x)$ of the same class, and

$$
\int_{0}^{2 \pi}|\bar{f}(x)|^{p} d x \leqq A_{p} \int_{0}^{2 \pi}|f(x)|^{p} d x
$$

holds, $A_{p}$ is a constant depending only on $p$.

(b) If the function $|f(x)| \log ^{+}|f(x)|$ is integrable, the conjugate of the Fourier series of $f(x)$ is the Fourier series of a function $\bar{f}(x)$ of the class $L$. Moreover, there exist two constants $A$ and $B$ such that

$$
\int_{0}^{2 \pi}|\bar{f}(x)| d x \leqq A \int_{0}^{2 \pi}|f(x)| \log ^{+}|f(x)| d x+B .
$$

In view of the importance of these theorems it may be of interest to give another proof of them based on a different idea. Actually it is

\footnotetext{
Received by the editors May 14, 1949.
} 
easy to see (and very well known) that they are equivalent to the following proposition about analytic functions.

Let $F(z)=u(z)+i v(z), u(z)>0, v(0)=0$ be a function regular inside the circle $|z|<1$. Then the inequalities

$$
\begin{aligned}
& \int_{0}^{2 \pi}\left|v\left(r e^{i x}\right)\right| p d x \leqq A_{p} \int_{0}^{2 \pi}\left|u\left(r e^{i x}\right)\right|^{p} d x ; \quad 1<p \leqq 2 ; r<1, \\
& \int_{0}^{2 \pi}\left|v\left(r e^{i x}\right)\right| d x \leqq C \int_{0}^{2 \pi}\left|u\left(r e^{i x}\right)\right| d x \\
& +D \int_{0}^{2 \pi} u \log u d x-2 \pi D u(0) \log u(0)
\end{aligned}
$$

hold, $C$ and $D$ being positive constants and $A_{p}$ the same as above.

Let us prove (1) first. The argument is based on the inequality

$$
|\sin \phi|^{p} \leqq A_{p}|\cos \phi|^{p}-B_{p} \cos \phi \phi
$$

valid for $-\pi / 2 \leqq \phi \leqq \pi / 2,1<p \leqq 2, A_{p}$ and $B_{p}$ being two positive constants depending on $p$.

The proof of it is simple. Since $\cos p \phi<0$ for $\phi= \pm \pi / 2$, taking $B_{p}$ large enough we get $-B_{p} \cos p \phi>1$ in a small neighborhood of $\phi= \pm \pi / 2$. Now $|\cos \phi|^{\dot{p}}$ is greater than a positive constant in every closed interval interior to $(-\pi / 2, \pi / 2)$, so that choosing $A_{p}$ large enough we shall have $A_{p}|\cos \phi|^{p}-B_{p} \cos p \phi>1$ in $(-\pi / 2, \pi / 2)$ and a fortiori the inequality (3).

To show (1) now, let us set

$$
F(z)=R e^{i \phi} ; \quad u=R \cos \phi ; \quad v=R \sin \phi .
$$

Owing to the fact that $u>0$, we may choose $-\pi / 2<\phi<\pi / 2$. For the same reason, $F(z) \neq 0$ and so $F(z)^{p}$ is regular in $|z|<1$. Integrating now along the circle $|z|=r$ we get

$\Re\left[\frac{1}{2 \pi i} \int_{C} \frac{F(z)^{p}}{z} d z\right]=\frac{1}{2 \pi} \int_{0}^{2 \pi} R^{p} \cos p \phi d x=\Re\left[F(0)^{p}\right]=u(0)^{p}>0$.

Multiplying now (3) by $R^{p}$ and integrating, we obtain

$$
\int_{0}^{2 \pi}\left|v\left(r e^{i x}\right)\right|^{p} d x \leqq A_{p} \int_{0}^{2 \pi}\left|u\left(r e^{i x}\right)\right|^{p} d x-B_{p} \int_{0}^{2 \pi} R^{p} \cos p \phi d x
$$

but since the last term is positive, we may drop it, and the desired result follows. A similar argument works in proving (2). Here instead of (3) we use 
(4)

$$
|\sin \phi| \leqq C \cos \phi+D(\cos \phi \log \cos \phi+\phi \sin \phi),
$$

which can be proved in exactly the same way.

Again, since $F(z)$ does not vanish in $|z|<1, F \log F$ is regular there, and integrating along $|z|=r$ we get

$\Re\left[\frac{1}{2 \pi i} \int_{C} F(z) \log F(z) \frac{d z}{z}\right]=\frac{1}{2 \pi} \int_{0}^{2 \pi} \Re[F \log F] d x=u(0) \log u(0)$, and replacing

$$
\begin{aligned}
\Re[F \log F] & =\Re[R(\cos \phi+i \sin \phi)(\log R \cos \phi-\log \cos \phi+i \phi)] \\
& =R \cos \phi \log R \cos \phi-R(\cos \phi \log \cos \phi+\phi \sin \phi),
\end{aligned}
$$

we obtain

$$
\begin{aligned}
\frac{1}{2 \pi} \int_{0}^{2 \pi} R(\cos \phi \log \cos \phi+\phi & \sin \phi) d x \\
& =\frac{1}{2 \pi} \int_{0}^{2 \pi} u \log u d x-u(0) \log u(0) .
\end{aligned}
$$

Finally, multiplying (4) by $R$ and integrating, we obtain

$$
\begin{aligned}
& \int_{0}^{2 \pi}\left|v\left(r e^{i x}\right)\right| d x \leqq C \int_{0}^{2 \pi}\left|u\left(r e^{i x}\right)\right| d x \\
& +D \int_{0}^{2 \pi} u \log u d x-2 \pi D u(0) \log u(0),
\end{aligned}
$$

as stated in (2).

\section{BIBLIOGRAPHY}

1. M. Riesz, Sur les fonctions conjuguees, Math. Zeit. vol. 27 (1927) pp. 218-244. 303.

2. A. Zygmund, Sur les fonctions conjuguées, Fund. Math. vol. 13 (1929) pp. 284-

3. —, Trigonometrical series, Warsaw, 1935.

University of Chicago 